\title{
A Decentralized Motion Coordination Strategy for Dynamic Target Tracking
}

\author{
Timothy H. Chung, Joel W. Burdick, Richard M. Murray \\ California Institute of Technology, Pasadena, CA 91125, USA \\ \{timothyc, jwb, murray\}@caltech.edu
}

\begin{abstract}
This paper presents a decentralized motion planning algorithm for the distributed sensing of a noisy dynamical process by multiple cooperating mobile sensor agents. This problem is motivated by localization and tracking tasks of dynamic targets. Our gradient-descent method is based on a cost function that measures the overall quality of sensing. We also investigate the role of imperfect communication between sensor agents in this framework, and examine the trade-offs in performance between sensing and communication. Simulations illustrate the basic characteristics of the algorithms.
\end{abstract}

\section{INTRODUCTION}

This paper considers the problem of decentralized mobile multi-agent motion control in the context of dynamic process estimation. We present a decentralized control law that is based on the local minimization of a cost function that assesses the quality of the sensing and estimation process. With this method, a team of mobile sensing agents can position themselves to provide the best overall estimation of a dynamical process. We also investigate how imperfect communication affects the coordination process. Practically, the goal of this work is to understand how sensing agent mobility can be used to improve the quality of sensing and estimation.

The work presented in this paper lies at the intersection of three fields: multi-robot coordination, sensor networks, and target tracking and estimation. In large part because of its relevance to military and civilian aeronautics, extensive work has already been done on the subjects of tracking targets with fixed sensors [1] or the optimal placement of fixed sensors [2]. However, sensor mobility has not been exploited in the classical literature on these subjects in order to improve estimation performance.

The study of multi-robot cooperation has recently received considerable attention. While many different approaches for cooperative motion-planning in a decentralized architecture have been developed, they are often tailored to specific applications, such as formation control for vehicle coordination using game-theoretic notions [3], cooperative object relocation via potential functions [4], mapping and exploration [5], search-and-rescue, and coverage and coordination tasks [6].

More recently, estimation techniques (see [7] for a survey) implementing distributed versions or approximations of the Kalman filter have been proposed. However, when control of the multiple sensor trajectories is considered, the motion control is computed using numerical techniques, whereas the decentralized motion control law presented in this paper is a closed-form, analytic solution governed by the structure of the distributed sensing problem.
The main contributions of this paper include the general formulation and closed-form analytical expressions for a decentralized motion control law for distributed sensing systems. In particular, each sensing agent governs its own motion while cooperating with other agents to reduce their combined uncertainty. We also provide preliminary results on how communication constraints can be incorporated into this framework.

The paper is organized as follows. Section II formulates the problem in which a team of sensing agents must estimate a dynamical system's state under uncertainty. We motivate the formulation with the task of dynamic target tracking. However, the methodology is not restricted to tracking applications. Section III investigates a decentralized motion control law that seeks to reduce the sensing team's collective estimate uncertainty via the use of mobility. We then develop an analogous decentralized control law in the presence of imperfect communication between the mobile agents in Section IV. Section V concludes the paper with a discussion of results and future work. Simulations illustrate the basic characteristics of our approach.

\section{PRoblem Formulation}

We are concerned with the problem of estimating the state $\mathbf{x}[k] \in \mathbb{R}^{n}$ of a general dynamical process:

$$
\mathbf{x}[k+1]=\mathbf{F} \mathbf{x}[k]+\mathbf{w}[k],
$$

where $\mathbf{F} \in \mathbb{R}^{n \times n}$ represents a linearized model of the dynamics and $k$ is the discrete time index. The process noise is denoted $\mathbf{w}[k] \in \mathbb{R}^{n}$ with the standard assumptions of $\mathbf{w}[k]$ being zero-mean, white and Gaussian, with process noise covariance matrix $\mathbf{Q}[k]$.

Suppose $M$ cooperating mobile sensors take measurements of this system. The observation made by the $i^{t h}$ mobile sensor, denoted $\mathbf{y}_{i}[k] \in \mathbb{R}^{m}$, is assumed to be given by the measurement model:

$$
\mathbf{y}_{i}[k]=\mathbf{H}_{i} \mathbf{x}[k]+\mathbf{v}_{i}[k],
$$

where $\mathbf{H} \in \mathbb{R}^{m \times n}$ is the measurement matrix, and the measurement noise $\mathbf{v}_{i}[k] \in \mathbb{R}^{m}$ for the $i^{t h}$ sensor is also assumed zero-mean, white and Gaussian, with covariance $\mathbf{R}_{i}[k] \in \mathbb{R}^{m \times m}$. Further, we assume that the measurement noise processes of the different sensors are independent.

The objective for the team of $M$ sensors is to configure themselves spatially in order to attain the best estimate of the process state from their combined observations. 


\section{A. Measurement Uncertainty Model}

The uncertainty in the $i^{t h}$ sensor's measurement is represented by the covariance matrix $\mathbf{R}_{i}$. For illustration, we assume the mobile agents can sense the range and bearing to the object being tracked. However, note that more general state-sensing paradigms can be used in this approach. In keeping with standard range-finding sensor models [8], the covariance matrix in this case has the following diagonal structure:

$$
\mathbf{R}_{i} \sim\left[\begin{array}{lr}
\left(\sigma_{\text {range }}^{i}\right)^{2} & 0 \\
0 & \left(\sigma_{\text {bearing }}^{i}\right)^{2}
\end{array}\right] .
$$

The variance of the range measurement noise is denoted by $\left(\sigma_{\text {range }}^{i}\right)^{2}$. The spatial dependence of the range uncertainty is represented by a function $f_{r}\left(r_{i}\right)$ of the distance $r_{i}$ from sensor $i$ to the target. The bearing noise variance $\left(\sigma_{\text {bearing }}^{j}\right)^{2}$ can also be modeled as dependent on the range, given by the function $f_{b}\left(r_{i}\right)$. Denoting the rotation matrix needed to transform the measurement noise covariance matrix, $\overline{\mathbf{R}}_{i}$, from local (i.e. range and bearing) coordinates to the global (Cartesian) reference frame as

$$
\mathbf{T}_{i}=\left[\begin{array}{cc}
\cos \left(\theta_{i}\right) & -\sin \left(\theta_{i}\right) \\
\sin \left(\theta_{i}\right) & \cos \left(\theta_{i}\right)
\end{array}\right],
$$

we obtain the measurement covariance model in global coordinates:

$$
\mathbf{R}_{i}[k] \triangleq \mathbf{T}_{i} \overline{\mathbf{R}}_{i}[k] \mathbf{T}_{i}^{T}=\mathbf{T}_{i}\left[\begin{array}{lr}
f_{r}\left(r_{i}\right) & 0 \\
0 & f_{b}\left(r_{i}\right)
\end{array}\right] \mathbf{T}_{i}^{T} .
$$

\section{B. Sensor Fusion}

The question of combining measurements from a team of mobile sensors is that of sensor fusion. Using a centralized method, such as a Kalman filter, with all observations would be computationally expensive, and would also counter our desire to have a decentralized solution. Instead, individual estimates of the target state and their covariances are exchanged and combined to yield a global estimate of the state in question. The measurements are combined using the following simple but general fusion relations [9]

$$
\mathbf{P}_{\text {fused }}^{-1} \hat{\mathbf{x}}=\sum_{i}^{M} \mathbf{P}_{i}^{-1} \hat{\mathbf{x}}_{i}, \quad \mathbf{P}_{\text {fused }}^{-1}=\sum_{i}^{M} \mathbf{P}_{i}^{-1},
$$

where $\hat{\mathbf{x}}_{i}$ and $\mathbf{P}_{i}, i=1, \ldots, M$ are the local estimate and estimate error covariance matrices, $\mathbf{P}_{\text {fused }}$ is the fused estimate error covariance matrix, and $\hat{\mathbf{x}}$ is the fused estimate of the target position. Note that the time index $k$ is implied.

We propose that the cost function $J$ to be minimized (by the motions of the mobile agents) is the determinant of the fused uncertainty matrix, $\mathbf{P}_{\text {fused }}$. Known as $D$ optimal design [10], the determinant is chosen (instead of the trace, maximum eigenvalue, etc.) to facilitate the analysis presented in Section III.

We investigate two methods to produce fused estimates: a simple scheme based on the fusion of local observations, and a scheme that combines local Kalman filter estimates. The first scheme requires minimal computation on-board each agent, while the local Kalman filter scheme takes advantage of the memory and predictive qualities of the Kalman filter, at the expense of greater complexity in computation.
Fusion of Local Observations: The fusion equation for the estimate error covariance in the case of shared observations becomes:

$$
\mathbf{P}_{\text {fused }}^{-1}=\sum_{i}^{M} \mathbf{R}_{i}^{-1}=\sum_{i}^{M}\left(\mathbf{T}_{i} \overline{\mathbf{R}}_{i} \mathbf{T}_{i}^{T}\right)^{-1}
$$

and hence, the cost function of interest is given by:

$$
J_{1}=\operatorname{det} \mathbf{P}_{\text {fused }}=\operatorname{det}\left(\sum_{i}^{M} \mathbf{R}_{i}^{-1}\right)^{-1} .
$$

Fusion of Locally Filtered Estimates: Alternatively, sensor observations can be processed locally by each mobile sensor using a Kalman filter to reduce the effect of uncertainty in measurements. The filtered result (i.e. the state estimate and estimate error covariance) is then shared and fused with those of other sensor nodes.

Recall the general Kalman filter equations,

$$
\begin{aligned}
\hat{\mathbf{x}}[k]^{-} & =\mathbf{F} \hat{\mathbf{x}}[k-1], \\
\mathbf{P}[k]^{-} & =\mathbf{F P}[k-1] \mathbf{F}^{T}+\mathbf{Q}, \\
\mathbf{K}[k] & =\mathbf{P}[k]^{-} \mathbf{H}^{T}\left(\mathbf{H P}[k]^{-} \mathbf{H}^{T}+\mathbf{R}\right)^{-1}, \\
\hat{\mathbf{x}}[k] & =\hat{\mathbf{x}}_{k}^{-}+\mathbf{K}[k]\left(\mathbf{y}[k]-\mathbf{H} \hat{\mathbf{x}}[k]^{-}\right), \\
\mathbf{P}[k] & =(\mathbf{I}-\mathbf{K}[k] \mathbf{H}) \mathbf{P}[k]^{-},
\end{aligned}
$$

where $\mathbf{K}$ is the Kalman estimator gain.

Thus, the $i^{t h}$ sensor generates its local estimate, $\hat{\mathbf{x}}_{i}$, and estimate error covariance,

$$
\mathbf{P}_{i}[k]=\boldsymbol{\Delta}_{i}-\mathbf{K}_{i} \mathbf{H}_{i} \boldsymbol{\Delta}_{i},
$$

where $\boldsymbol{\Delta}_{i} \triangleq \mathbf{P}_{i}[k]^{-}=\mathbf{F P}_{i}[k-1] \mathbf{F}^{T}+\mathbf{Q}$, and exchanges these quantities with the other sensing agents. Note that $\Delta$ is independent of current sensor locations, whereas the Kalman gain $\mathbf{K}_{i}$ depends upon the observation location through the dependence of the measurement noise covariance matrix $\mathbf{R}_{i}$ on range to the target.

The cost function, $J_{2}$, to be minimized in this case is again the determinant of $P_{\text {fused }}$ :

$$
J_{2}=\operatorname{det} \mathbf{P}_{\text {fused }}=\operatorname{det}\left(\sum_{i}^{M}\left(\boldsymbol{\Delta}_{i}-\mathbf{K}_{i} \mathbf{H}_{i} \boldsymbol{\Delta}_{i}\right)^{-1}\right)^{-1} \text {. }
$$

Note that $J_{1}$ and $J_{2}$ are functions of the target and sensor positions due to the spatial dependence intrinsic in the measurement noise covariance. Thus by varying the positions of the sensors, we can influence the estimate error covariance. The problem we pose is how to do so in a decentralized way.

\section{GRAdient AnALysis}

This section presents an approach to minimize the cost in a gradient-descent-based manner. In essence, we seek to answer to the question, Where should the $l^{\text {th }}$ sensor move in order to reduce sensing uncertainty? The motivation for using the gradient is its decentralizing effect on functions that can be represented as sums, which are of similar form as (2) and (3). Proofs of convergence to local minima for gradient-based methods can be found in [11].

To compute the gradients of $J_{1}$ and $J_{2}$, we employ the following standard matrix calculus identities for the chain 
rule, derivative of the determinant, and derivative of the inverse [12], respectively:

$$
\begin{aligned}
\frac{\partial}{\partial z} h(\mathbf{A}(z)) & =\operatorname{tr}\left[\frac{\partial h}{\partial \mathbf{A}} \frac{\partial \mathbf{A}}{\partial z}\right] \\
\frac{\partial}{\partial \mathbf{A}} \operatorname{det}(\mathbf{A}) & =|\mathbf{A}| \mathbf{A}^{-T}=|\mathbf{A}| \mathbf{A}^{-1} \\
\frac{\partial}{\partial z} \mathbf{A}^{-1} & =-\mathbf{A}^{-1}\left(\frac{\partial \mathbf{A}}{\partial z}\right) \mathbf{A}^{-1}
\end{aligned}
$$

where $\mathbf{A} \in \mathbb{R}^{n \times n}$ is a symmetric, positive-definite matrix, $h: \mathbb{R}^{n \times n} \rightarrow \mathbb{R}$ is a real-valued matrix function, and $z \in \mathbb{R}^{n}$ is a scalar variable.

The cost functions can be represented as a function of polar variables, where the gradient in polar coordinates is:

$$
\nabla J\left(r_{1}, \theta_{1}, \ldots, r_{M}, \theta_{M}\right)=\sum_{i}^{M}\left(\frac{\partial J}{\partial r_{i}} e_{r_{i}}+\frac{1}{r_{i}} \frac{\partial J}{\partial \theta_{i}} e_{\theta_{i}}\right)
$$

where $e_{r}$ and $e_{\theta}$ are the radial and tangential unit vectors.

Accordingly, we seek to compute the derivatives of the cost function with respect to the $l^{\text {th }}$ sensor's state in order to determine the locally cost-minimizing path for sensor $l$.

Proposition 1: The gradient of $J_{1}$ and $J_{2}$ with respect to the $l^{\text {th }}$ sensor's coordinate $z_{l}$ (where $z_{l}$ represents either $r_{l}$ or $\theta_{l}$ ) relative to the target has the general form:

$$
\begin{gathered}
\frac{\partial J}{\partial z_{l}}=\left|\mathbf{P}_{\text {fused }}\right| \operatorname{tr}\left[\boldsymbol{\Pi} \frac{\partial \mathbf{R}_{l}}{\partial z_{l}} \boldsymbol{\Pi}^{T} \mathbf{P}_{\text {fused }}\right], \\
\text { where } \quad \boldsymbol{\Pi}=\left\{\begin{array}{cl}
\mathbf{R}_{l}^{-1}, & \text { for } J_{1}, \\
\mathbf{P}_{l}^{-1} \mathbf{K}_{l}, & \text { for } J_{2} .
\end{array}\right.
\end{gathered}
$$

In terms of range and bearing coordinates, $r_{l}$ and $\theta_{l}$, respectively, we have

$$
\begin{aligned}
& \frac{\partial \mathbf{R}_{l}}{\partial r_{l}}=\frac{\partial}{\partial r_{l}} \mathbf{T}_{l} \overline{\mathbf{R}}_{l} \mathbf{T}_{l}^{T}=\mathbf{T}_{l}\left[\begin{array}{cc}
\frac{\partial f_{r}}{\partial r_{l}} & 0 \\
0 & \frac{\partial f_{b}}{\partial r_{l}}
\end{array}\right] \mathbf{T}_{l}^{T}, \text { and } \\
& \frac{\partial \mathbf{R}_{l}}{\partial \theta_{l}}=\frac{\partial}{\partial \theta_{l}} \mathbf{T}_{l} \overline{\mathbf{R}}_{l} \mathbf{T}_{l}^{T}=\Psi \mathbf{T}_{l} \overline{\mathbf{R}}_{l} \mathbf{T}_{l}^{T}+\mathbf{T}_{l} \overline{\mathbf{R}}_{l} \mathbf{T}_{l}^{T} \Psi^{T}
\end{aligned}
$$

where $\quad \Psi=\left[\begin{array}{cc}0 & -1 \\ 1 & 0\end{array}\right]$.

Proof: Proof is realized by application of (4)-(6) to either (2) or (3).

\section{A. Gradient-descent Control Law}

The gradient formulas can be used to define a control law, $\mathbf{u}_{l}\left(r_{l}, \theta_{l}\right)$, for the motion of each sensor. The direction that the $l^{\text {th }}$ sensor should move to achieve the steepest descent of the cost function is given by the gradient:

$$
\nabla_{r_{l}, \theta_{l}} J\left(r_{1}, \theta_{1}, \ldots, r_{M}, \theta_{M}\right)=\frac{\partial J}{\partial r_{l}} e_{r_{l}}+\frac{1}{r_{l}} \frac{\partial J}{\partial \theta_{l}} e_{\theta_{l}} .
$$

Hence, the gradient-based control law for the cost function given by either (2) or (3) is

$$
\mathbf{u}_{l}\left(r_{l}, \theta_{l}\right)=\left[\begin{array}{ll}
\left(\frac{\partial J}{\partial r_{l}}\right) & \frac{1}{r_{l}}\left(\frac{\partial J}{\partial \theta_{l}}\right)
\end{array}\right]^{T},
$$

and due to the decentralizing effect of the gradient on the cost function, $\mathbf{u}_{l}\left(r_{l}, \theta_{l}\right)$ is an explicit function of only the $l^{\text {th }}$ sensor's position. In other words, the $l^{t h}$ sensor's motion control law can be computed from its current state, its sensor model and the fused estimate covariance. The remaining task is to convert the control signal from polar coordinates to Cartesian coordinates,

$$
\mathbf{u}_{l}\left(r_{l}, \theta_{l}\right)=\mathbf{T}_{l} \mathbf{u}_{l}\left(x_{l}, y_{l}\right) \Rightarrow \mathbf{u}_{l}\left(x_{l}, y_{l}\right)=\mathbf{T}_{l}^{T} \mathbf{u}_{l}\left(r_{l}, \theta_{l}\right)
$$

Using this decentralized control law, the sensors maneuver themselves into a local configuration which yields the highest sensing quality, given their particular sensor models.

As an illustrative example, consider a measurement uncertainty model where the measurement noise in range is quadratic in the distance to target $j$. In other words, let $f_{r_{j}}\left(r_{j}\right)=a_{2}\left(r_{j}-a_{1}\right)^{2}+a_{0}$, where $a_{0}, a_{1}, a_{2}$ are constant coefficients. This model corresponds to the notion of a "sweet spot" in sensing, located at a distance $a_{1}$ from the target, where uncertainty in measurements is minimal [13]. Furthermore, let the measurement noise in bearing simply be a fixed multiple $\alpha$ of the range noise variance, such that $f_{b_{j}}\left(r_{j}\right)=\alpha f_{r_{j}}\left(r_{j}\right)$.

The resulting configuration, for the case of a team of homogeneous sensors (i.e. $f_{r_{j}}=f_{r}, \forall_{j}$ ), with $a_{0}=$ $0.1528, a_{1}=15.625, a_{2}=0.0008$, is depicted in Fig. 1, where the motion of the agents are governed by cost functions $J_{1}$ and $J_{2}$ (shown in (a) and (b), respectively).
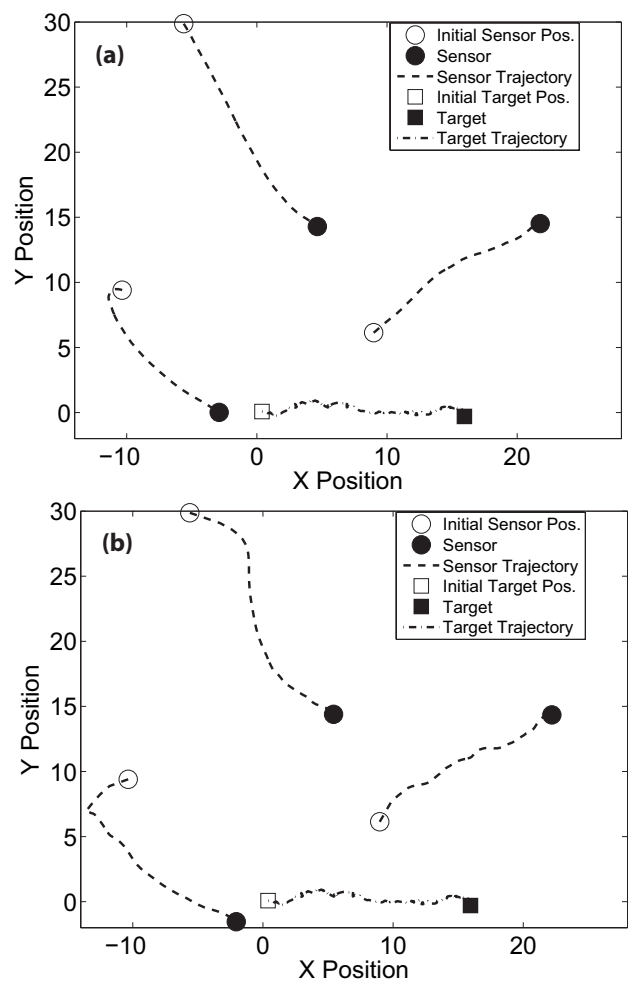

Fig. 1. Three Sensors Track a (Biased) Randomly-Walking Target using the Proposed Gradient-Descent Control Law Using Costs (a) $J_{1}$, (b) $J_{2}$.

The sensors converge to an intuitively symmetric configuration, where the three similar sensors are uniformly distributed at the optimal sensing distance from the target.

A comparison of the performance under the two cost functions is shown in Fig. 2, and is contrasted to the scenario where fixed sensors are tracking the same moving target. Clearly, mobility aids in reducing the uncertainty in the state estimate. Further, as expected, the local processing of measurements provides significant improvement over simply sharing observations, due to the filter's ability to incorporate previous measurements and predict target motion. However, if on-board computation is limited, motion control based on 


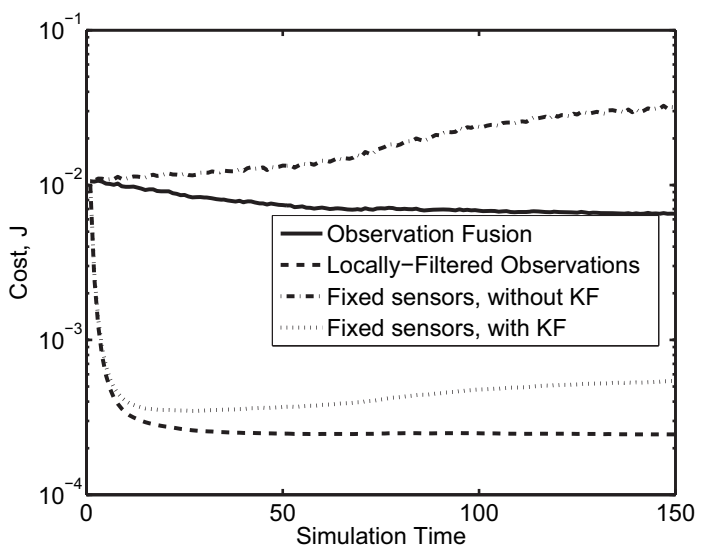

Fig. 2. Comparison of the algorithms: Evolution of the cost

simple observation fusion may serve as a less computationally intensive alternative.

We can further compare the performance of the proposed scheme with the approach presented in [13]. In that previous work, the authors approximate the gradient of the cost expression with a search over a discrete set of local motions. The discrete search methodology requires each sensor to predict future estimates of the target location for all sensors, propagate the effect of movement on the predicted global performance, and then choose the cost-minimizing action. Fig. 3 illustrates the trajectories of three homogeneous sensors using the approach presented in [13], given the same initial conditions as shown in Fig. 1.

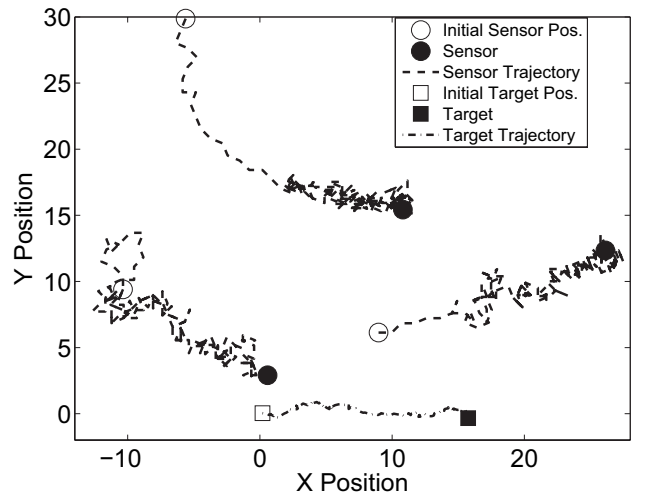

Fig. 3. Three Sensors Track a (Biased) Randomly-Walking Target using the Discrete-Search Method in [13].

The discrete-search method results in jittery trajectories, partly due to the fixed step size and partly due to the algorithm's sensitivity to noise in the target motion. More specifically, the optimality of each sensor's actions is limited by the resolution of the discrete search space. In contrast, our proposed control law is the best instantaneous cost-minimizing action. Prediction of other sensors' estimates/covariances is no longer necessary, as only local information for each sensor is required to generate the control signal. Efficient matrix manipulation algorithms and closed-form gradient formulae further improve the computational efficiency of this gradient-based approach. Also, while gradient-based methods only guarantee convergence to local minima, we find that the sensing performance of the mobile sensing

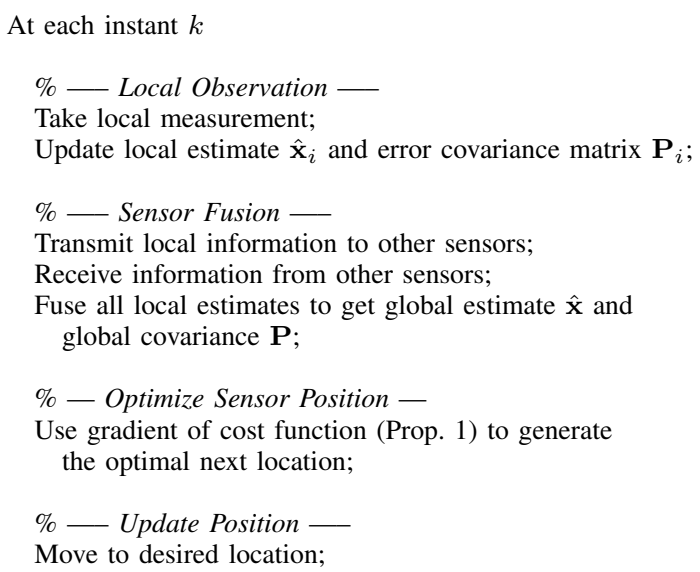

TABLE I

PSEUdo-COde of Motion Control Strategy

team, even at local minima of the cost function, is generally greater than the case of fixed sensors. The proposed motioncontrol algorithm is summarized in Table I.

\section{B. Heterogeneous Sensing Uncertainty Models}

This section considers a more general situation where the team comprises heterogeneous sensors. As a simple example, assume that the sensors have a quadratic uncertainty model, but with different coefficients (i.e. "different sweet spots"). Hence, let the $j^{\text {th }}$ sensor's uncertainty model be

$$
f_{r_{j}}\left(r_{j}\right)=a_{2}^{j}\left(r_{j}-a_{1}^{j}\right)^{2}+a_{0}^{j}, \quad f_{b_{j}}\left(r_{j}\right)=\alpha^{j} f_{r_{j}}\left(r_{j}\right),
$$

The resulting configuration of three sensors with varying sensor models is shown in Fig. 4.

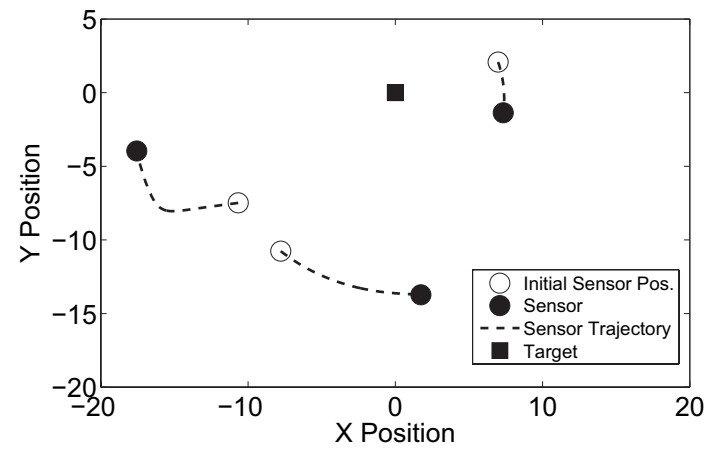

Fig. 4. Decentralized Motion Control with Heterogeneous Sensor Uncertainty Models

The generalization of the motion control law to varying sensor types is straightforward, and is a direct consequence of the structure of the cost function and its gradient-based decentralization. As can be expected, symmetries in the resulting sensor configuration are broken due to the sensors' heterogeneity. However, the sensors still achieve a costminimizing configuration without requiring explicit knowledge of other sensors' measurement models.

\section{Observations of Multiple Targets}

The gradient formulation readily extends to the case where multiple targets are observed simultaneously. By augmenting the multiple observation vectors and assuming that measurements of distinct targets are independent and appropriately 
associated, similar expressions for the gradient as (7) can be produced, redefining $\overline{\mathbf{R}}, \mathbf{T}$, and $\mathbf{P}$ as the following block matrices:

$$
\begin{gathered}
\overline{\mathbf{R}}_{i} \rightarrow \operatorname{diag}\left(\overline{\mathbf{R}}_{i}^{1}, \ldots, \overline{\mathbf{R}}_{i}^{N}\right), \mathbf{T}_{i} \rightarrow \operatorname{diag}\left(\mathbf{T}_{i}^{1}, \ldots, \mathbf{T}_{i}^{N}\right), \\
\mathbf{P} \rightarrow \operatorname{diag}\left(\mathbf{P}^{1}, \ldots, \mathbf{P}^{N}\right),
\end{gathered}
$$

for $N$ simultaneously-observed targets, such that $\overline{\mathbf{R}}_{i}, \mathbf{T}_{i}, \mathbf{P} \in \mathbb{R}^{n N \times n N}$. The specifics of data and track association are beyond the scope of this paper. See [9] for relevant methods.

Proposition 2: The gradient of $J_{1}$ and $J_{2}$ with respect to the $l^{\text {th }}$ sensor's coordinate $z_{l}^{j}$ relative to the $j^{\text {th }}$ target has the general form given by:

$$
\begin{gathered}
\frac{\partial J}{\partial z_{l}^{j}}=\left|\mathbf{P}_{\text {fused }}\right| \operatorname{tr}\left[\boldsymbol{\Pi} \frac{\partial \mathbf{R}_{l}^{j}}{\partial z_{l}^{j}} \mathbf{\Pi}^{T} \mathbf{P}_{\text {fused }}\right], \\
\boldsymbol{\Pi}=\left\{\begin{array}{cl}
\left(\mathbf{R}_{l}^{j}\right)^{-1}, & \text { for } J_{1}, \\
\left(\mathbf{P}_{l}^{j}\right)^{-1} \mathbf{K}_{l}^{j}, & \text { for } J_{2} .
\end{array}\right.
\end{gathered}
$$

where

Since the cost is now a function of additional variables, its gradient is given by:

$$
\nabla J(\cdot)=\sum_{j}^{N} \sum_{i}^{M}\left(\frac{\partial J}{\partial r_{i}^{j}} e_{r_{i}^{j}}+\frac{1}{r_{i}^{j}} \frac{\partial J}{\partial \theta_{i}^{j}} e_{\theta_{i}^{j}}\right) .
$$

The modified control law for the $l^{t h}$ sensor observing $N$ targets is given by:

$$
\mathbf{u}_{l}\left(r_{l}, \theta_{l}\right)=\sum_{j}^{N} \mathbf{u}_{l}^{j}\left(r_{l}^{j}, \theta_{l}^{j}\right),
$$

where $\mathbf{u}_{l}^{j}=\left[\left(\frac{\partial J}{\partial r_{l}^{j}}\right), \frac{1}{r_{l}^{j}}\left(\frac{\partial J}{\partial \theta_{l}^{j}}\right)\right]^{T}, j=1, \ldots, N$. An example of the resulting multi-target tracking performance is illustrated in Fig. 5.

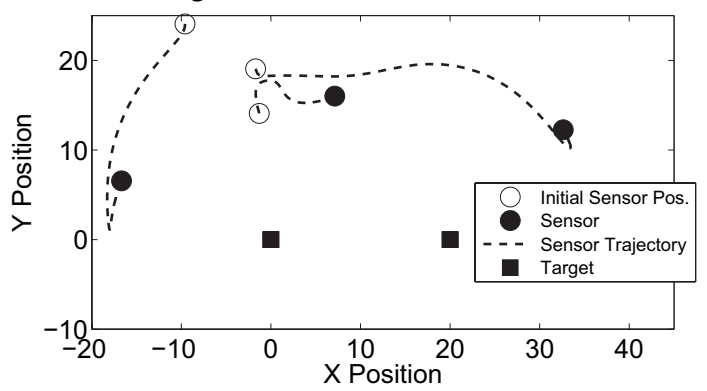

Fig. 5. Three Sensors Track Two Targets

Sensor Teams: It is often likely that all targets cannot be simultaneously observed by each sensor. Known as the sensor assignment problem, the difficult task of assigning particular sensors to specific targets is an active area of research. As simply a means of demonstrating the feasibility of integrating sensor assignment tasks with the proposed tracking algorithm, we employ a simple round-robin strategy [14]. An incomplete, but illustrative, list of references on the assignment problem is [15], [16], [17].

Round-robin assignment is done by arbitrarily ordering the sensing agents. Sensor 1 (S1) first computes the target with the largest estimate uncertainty. S1 selects this target (T1, as depicted in Fig 6), announces its assignment, and employs the local gradient-based control law to optimize sensing performance. Sensor 2 (S2) chooses its assignment from the remaining targets (namely, T2), and so on. If sensors outnumber targets $(M>N)$, remaining sensors are iteratively assigned to targets with greatest uncertainty. If $M<N$, the sensors will make their assignments to minimize the effect of the unobserved targets. Further, each sensor stores its previous assignments so as to prevent erratic assignment switching between targets.
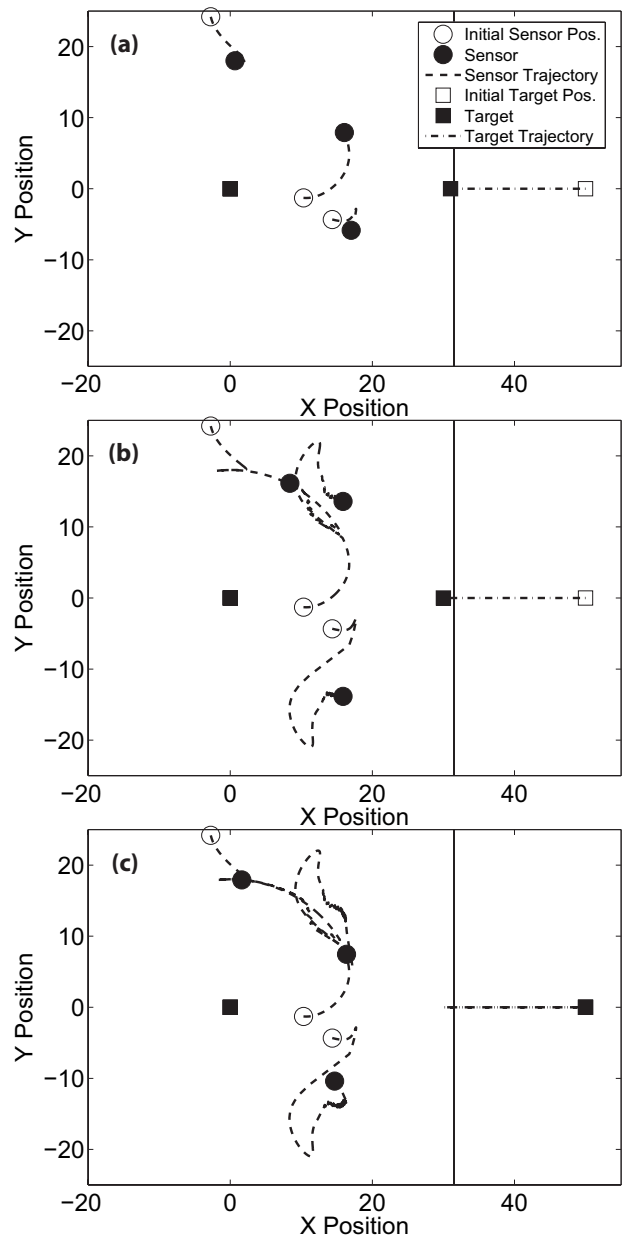

Fig. 6. Simulation of sensor assignment implementation: (a) Three sensors observe T1, (b) Once T2 crosses threshold, S1 and S3 maneuver to observe $\mathrm{T} 2$, and (c) Three sensors regroup (after T2 leaves region) to observe T1.

\section{IMPERFECT COMMUNICATION}

When the communication channel between sensing agents is imperfect, where the noise in communication depends on transmission distances, there exists a tradeoff between motion to improve sensing performance and motion to improve communication quality.

The homogeneous sensor observation model can be easily modified to include the effects of noisy communication links, such as fading wireless channels. The transmission of the $i^{t h}$ sensor's observation $\mathbf{y}_{i}$ received at sensor $j$ is:

$$
\hat{\mathbf{y}}_{j, i}[k]=\mathbf{y}_{i}[k]+\mathbf{v}_{j, i}^{c}[k]=\mathbf{H x}[k]+\mathbf{v}_{i}[k]+\mathbf{v}_{j, i}^{c}[k],
$$

where $\mathbf{v}_{j, i}^{c}[k] \in \mathbb{R}^{m}$ is the communication noise in the received observation. We assume that the communication noise across links is symmetric, i.e. $\mathbf{v}_{j, i}^{c}=\mathbf{v}_{i, j}^{c}$, and that there is no noise for self-transmissions. 
Denote the covariance of the communication noise vector, $\mathbf{v}_{j, i}^{c}$ as $\mathbf{C}_{j, i} \in \mathbb{R}^{m \times m}$. The spatial dependence of this noise is often related to issues such as transmission power or environmental effects. Letting $d_{i, j}$ denote the distance between sensor agents $i$ and $j$, we pose a general model for the covariance of the communication noise:

$$
\mathbf{C}_{j, i}=\left[\begin{array}{cc}
g_{j, i}\left(d_{j, i}\right) & 0 \\
0 & g_{j, i}\left(d_{j, i}\right)
\end{array}\right],
$$

where $g_{j, i}\left(d_{j, i}\right)$ represents the communication noise uncertainty's dependence on the inter-agent distance $d_{i, j}$.

Given the measurement and communication channel models, the modified fusion equation for the covariance of the estimate error is [18]:

$$
\mathbf{P}_{j, f \text { used }}^{-1}=\sum_{i}^{M}\left(\mathbf{P}_{i}+\mathbf{C}_{j, i}\right)^{-1}
$$

where $\mathbf{P}_{j, \text { fused }}$ is the $j^{\text {th }}$ sensor's fused estimate error covariance matrix. Note that due to the communication noise, $\mathbf{P}_{j, \text { fused }}$ will likely differ between sensors.

In this case, the cost function $J_{3, j}$ to be minimized is the determinant of the fused uncertainty matrix, given as:

$$
J_{3, j}=\operatorname{det} \mathbf{P}_{j, f \text { used }}=\operatorname{det}\left(\sum_{i}^{M}\left(\mathbf{P}_{i}+\mathbf{C}_{j, i}\right)^{-1}\right)^{-1} .
$$

Similar to $J_{1}$ and $J_{2}$, this cost function is also dependent on the (relative) positions of the sensors with respect to the target and one another, noting that the inter-sensor distance $d_{j, i}$ is implicitly a function of $\left(r_{i}, \theta_{i}, r_{j}, \theta_{j}\right)$.

Following the techniques of the previous section yields the following results:

Proposition 3: The gradient of $J_{3, l}$ with respect to the $l^{\text {th }}$ sensor's coordinate $z_{l}$ relative to the target is given by:

$$
\begin{aligned}
\frac{\partial J_{3, l}}{\partial z_{l}}= & \left|\mathbf{P}_{l, \text { fused }}\right| \operatorname{tr}\left[\boldsymbol{\Pi} \frac{\partial \mathbf{R}_{l}}{\partial z_{l}} \boldsymbol{\Pi}^{T} \mathbf{P}_{l, \text { fused }}\right] \\
& +\sum_{i \neq l}^{M}\left|\mathbf{P}_{l, \text { fused }}\right| \operatorname{tr}\left[\boldsymbol{\Phi}_{l, i} \frac{\partial \mathbf{C}_{l, i}}{\partial z_{l}} \boldsymbol{\Phi}_{l, i} \mathbf{P}_{l, \text { fused }}\right],
\end{aligned}
$$

where $\boldsymbol{\Phi}_{l, i} \triangleq\left(\mathbf{P}_{i}+\mathbf{C}_{l, i}\right), \boldsymbol{\Pi}$ is chosen according to whether simple observations (i.e. $\mathbf{P}_{l}=\mathbf{R}_{l}$ ) or locally-filtered measurements (i.e. $\mathbf{P}_{l}=\boldsymbol{\Delta}_{l}-\mathbf{K}_{l} \mathbf{H}_{l} \boldsymbol{\Delta}_{l}$ ) are fused, and

$$
\frac{\partial \mathbf{C}_{l, i}}{\partial z_{l}}=\left[\begin{array}{cc}
\frac{\partial g_{l, i}}{\partial z_{l}} & 0 \\
0 & \frac{\partial g_{l, i}}{\partial z_{l}}
\end{array}\right] \text {. }
$$

The sensors' motions are governed by two influences, namely that of moving to improve sensing quality combined with moving to improve communication performance. Note that these effects, in the context of the motion control signal, are decoupled. This point is examined more closely in the following section.

Many different models exist for the inter-nodal communication noise, which may include the effects of transmission distance and power, frequency, quantization sizes, and number of bits per transmission over the channel (see [18] and references therein). We choose a simple illustrative noise model, where the signal-to-noise ratio $(S N R)$ obeys an inverse-square law [19]:

$$
\sigma_{i, j}^{2}=\frac{1}{S N R}, \quad S N R=\frac{\kappa}{d_{i, j}^{2}},
$$

where $\kappa>0$ is a constant and $d_{i, j}$ is the distance between sensor $i$ and sensor $j$. For this model, the performance of the modified gradient-based control law for the task of target tracking is depicted in Fig. 7, with $\kappa=1000$. The resulting configuration of the sensors under communication constraints is shown overlaying the outcome under the same initial conditions without communication noise.

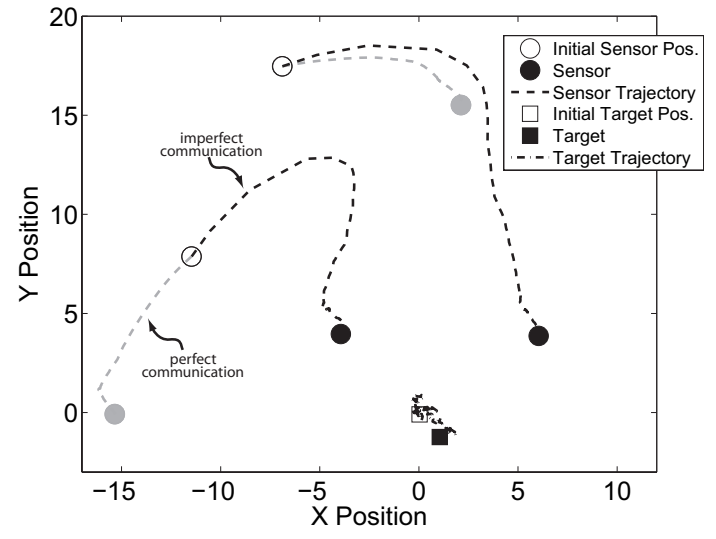

Fig. 7. Two Sensors Track Randomly-Walking Target: With Imperfect Communication (black), or With Perfect Communication (gray)

Clearly, the communication constraint biases the mobile agents to remain closer to one another. This behavior results in a compromise of sensing performance. Note that the sensing agents maintain their relative bearing for optimal sensing, given the above sensing and communication models. Different communication models may yield configurations where the agents maintain their optimal sensing distances and instead reduce their relative bearing to the target [18].

\section{A. Relationship between Sensing and Communication Per- formance Objectives}

Next we can examine how performance, measured by the cost function (11), is related to communication and sensing parameters. Varying the parameter $\kappa$ (which is an aggregate measure of communication characteristics) results in Fig. 8(a) for the case where multiple sensors observe a single target. As $\kappa$ becomes small (communication noise becomes large) no useful information is exchanged amongst the sensing agents. Thus, each agent tends to act independently, and none of the benefits of cooperative sensing is realized. For high values of $\kappa$ (the communication link quality is very good), overall cost is reduced by the use of an increasing number of sensors. Interesting behavior appears to occur in the intermediate range, where there is a tradeoff between the sensing and communication objectives.

Next we vary the optimal sensing distance (a.k.a. "sweet spot), $a_{1}$. As shown in Fig. 8(b), reducing the optimal range to a target has the effect of improving the overall system performance-the smaller this distance is, the closer the cooperating sensors can operate while maintaining a wide-enough perspective. Conversely, while large separations between sensors provide sufficiently different views of the target, communication noise increases commensurately. 

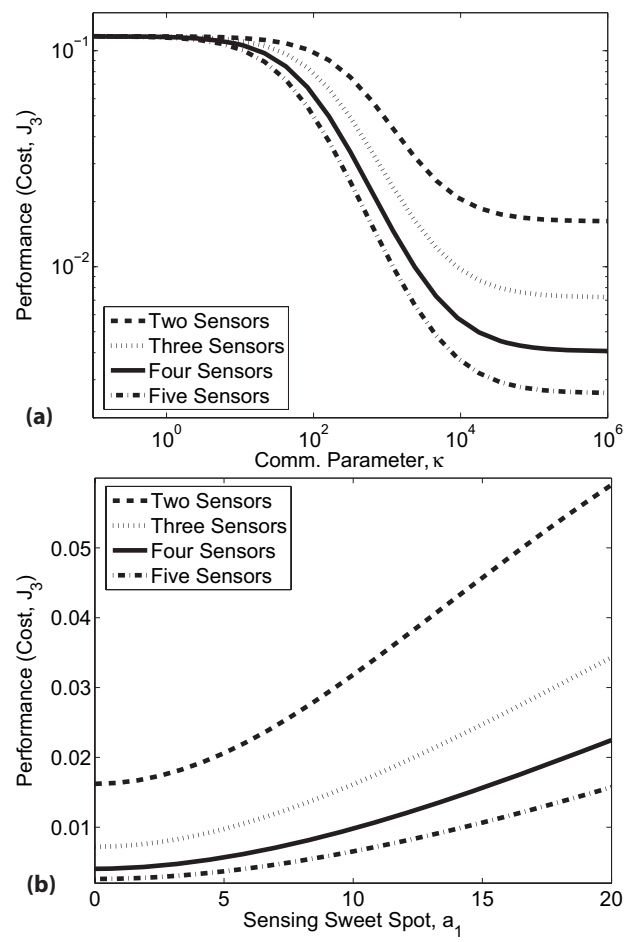

Fig. 8. Sensing and Communication Performance Tradeoff as a Function of (a) Communication Parameter, $\kappa$, (b) Sensing Sweet Spot, $a_{1}$

Clearly, the choice or design of sensors plays a role in the overall performance of the mobile sensing network.

\section{Discussion AND Future WORK}

We proposed a gradient-based decentralized motion control law for a team of mobile agents, equipped with (possibly heterogeneous) sensors and taking measurements of (possibly multiple) dynamical processes, embodied in this work as moving targets. Sensor mobility is used to improve estimates, as measurement noise is spatially-dependent on sensor positions. The specific formulae for the gradients results in an efficient implementation. Using a simple sensor assignment rule, we also examined the formation of subgroups of sensors to manage the distributed task. We then investigated the effect of a noisy communication environment, where disturbances also depend on inter-agent distances, and developed a modified motion control law. Further, motivated by the decoupling nature of this decentralized control law, we examined the tradeoffs between sensing and communication and their dependence on sensing and communication parameters.

Immediate future work includes a thorough analysis of the computational complexity of this framework as a function of the number of sensors and/or targets. Similarly, we seek convergence properties of the motion control strategy in terms of estimation error bounds, and understanding the resulting steady-state sensor configurations for different classes of target motions.

Additionally, we can examine the proposed strategy under relaxations of the assumptions made. For example, the uncertainty profile of the sensor model or the communication link may not be known a priori, and hence collected and shared data can be used to determine these profiles in realtime. Further, fully-connected communication networks, in practice, are impractical; an additional area of future research is how different network topologies can be addressed.

Also, the use of more complex models for both the target and sensor agents is relatively straightforward and should be investigated. Other target-tracking methods, such as pursuitevasion concepts, offer approaches where targets actively try to confound the mobile sensors. This scenario requires that the sensor agents consider the target behavior beyond simply a dynamical motion model. Incorporation of these types of ideas may offer new perspectives on target-tracking and other distributed sensing applications.

\section{REFERENCES}

[1] Y. Bar-Shalom and X.-R. Li, Estimation and Tracking: Principles, Techniques, and Software. Norwood, MA: Artech House, Inc, 1993.

[2] D. Ucinski, Optimal Measurement Methods for Distributed Parameter System Identification. Boca Raton: CRC Press, 2005.

[3] G. Inalhan, D. M. Stipanovic, and C. Tomlin, "Decentralized optimization, with application to multiple aircraft coordination," Stanford University, Tech. Rep. SUDAAR-759, February 2003.

[4] G. Pereira, M. Campos, and V. Kumar, "Decentralized algorithms for multi-robot manipulation via caging," Intl. Journal of Robotics Research, vol. 23, no. 7-8, pp. 783-795, 2004.

[5] S. Roumeliotis and G. Bekey, "Distributed multi-robot localization," Trans. on Robotics and Automation, vol. 18, no. 5, pp. 781-795, 2002

[6] F. Bullo and J. Cortes, Cooperative Control, ser. Lecture Notes in Control and Information Sciences. New York: Springer Verlag, 2005, vol. 309, ch. Adaptive and Distributed Coordination Algorithms for Mobile Sensing Networks, pp. 43-62.

[7] N. G. Wah and Y. Rong, "Comparison of decentralized tracking algorithms," in Proc. of Intl. Conf. on Info. Fusion, 2003, pp. 107-113.

[8] K. Ramachandra, Kalman Filtering Techniques for Radar Tracking. New York, NY: Marcel Dekker, Inc., 2000.

[9] Y. Bar-Shalom and T. E. Fortman, Tracking and Data Association. Academic Press, 1988.

[10] L. Mihaylova, T. Lefebvre, H. Bruyninckx, K. Gadeyne, , and J. D. Schutter, "Active sensing for robotics - a survey," in Proc. of the 5th Intl. Conf. on Numerical Methods and Applications, Borovets, Bulgaria, August 2002, pp. 316-324.

[11] D. Bertsekas and J. Tsitsiklis, Parallel and Distributed Computation : Numerical Methods. Prentice Hall, 1989.

[12] D. S. Bernstein, Matrix Mathematics. Princeton, NJ: Princeton University Press, 2005.

[13] T. H. Chung, V. Gupta, J. W. Burdick, and R. M. Murray, "On a decentralized active sensing strategy using mobile sensor platforms in a network," in Proc. of the IEEE Conf. on Decision and Control, Paradise Island, Bahamas, Dec 2004.

[14] T. Mukai and I. Ishikawa, "An active sensing method using estimated errors for multisensor fusion systems," in Proc. Intl. Conf. on Multisensor Fusion and Integ. for Intel. Sys., Oct 1994, pp. 615-622.

[15] W. L. Brogan, "Algorithm for ranked assignments with applications to multiobject tracking," Journal of Guidance, Control, and Dynamics, vol. 12, no. 3, pp. 357-364, 1989.

[16] V. Isler, S. Khanna, J. Spletzer, and C. Taylor, "Target tracking with distributed sensors: The focus of attention problem," Computer Vision and Image Understanding Journal, vol. Special Issue on Attention and Performance in Computer Vision, p. accepted, 2005, accepted.

[17] R. E. Burkard, "Selected topics on assignment problems," Discrete Applied Mathematics, vol. 123, no. 1-3, pp. 257 - 302, November 2002. [Online]. Available: http://www.opt.math.tu-graz.ac.at/burkard/

[18] Y. Mostofi, T. H. Chung, R. M. Murray, and J. W. Burdick, "Communication and sensing trade-offs in decentralized mobile sensor networks: A cross-layer design approach," in Intl. Conf. on Information Processing in Sensor Networks, 2005.

[19] J. D. Gibson, The Mobile Communications Handbook, 2nd ed. CRC Press and IEEE Press, 1999. 